\title{
METACHROMASIA AFTER TREATING TISSUE SECTIONS WITH SULPHURIC ACID
}

\author{
BY \\ H. KRAMER AND G. M. WINDRUM \\ From the Department of Pathology, Radcliffe Infirmary, Oxford
}

(RECEIVED FOR PUBLICATION MARCH 26, 1953)

The recent introduction by McManus and Mowry (1952) of a sulphuric acid-haematoxylin method for the demonstration of basement membrane prompted us to investigate the effects of pretreating histological sections with sulphuric acid on the capacity of tissue elements to strain metachromatically. Surprising as it may seem, adequately fixed and dehydrated tissues withstand five minutes' immersion in concentrated sulphuric acid without charring or showing any severe disturbance of their structure. Subsequent staining with a metachromatic dye reveals that a greatly increased range of tissue elements shows a metachromatic affinity, and this staining technique offers a new and useful polychrome method which may well have some degree of histochemical specificity.

The technique which has been developed involves immersion of sections in concentrated sulphuric acid, followed by staining with a dilute solution of a metachromatic dye. The method is simple, quick, and reliable.

\section{Method}

Any of the commonly used fixatives is satisfactory. (1) Paraffin sections are mounted on slides and left to dry thoroughly. (2) The paraffin wax is removed with xylene, and the section rinsed in absolute ethanol and allowed to dry. (3) The section is immersed in concentrated sulphuric acid (commercial sulphuric acid, S.G. 1.842). Sixty to seventy-five seconds is sufficient, and longer exposure to the acid increases the risk of detaching sections from the slides. (4) The section is washed thoroughly in running tap-water. (5) It is stained for five minutes in $0.01 \%$ " azur A," in $30 \%$ ethanol (Azur A Revector, Hopkin \& Williams Ltd., London). (6) It is then rinsed in $70 \%$ ethanol, dehydrated in two changes of absolute ethanol (approximately two minutes in each), cleared in xylene, and mounted in D.P.X.

There is some tendency for sections to become detached from the slides during stages 3 or 4 . Thin, flat sections which have been thoroughly dried generally cause little difficulty, but poor preparations, particularly of rather friable tissues such as muscle or skin, may be troublesome. This difficulty can be largely overcome by coating the sections with a watersoluble wax before immersing them in the acid. The sections are dipped twice in a saturated solution of ester wax in acetone and allowed to dry, leaving them thinly coated with the wax. Hydrowax " $\mathrm{H}$ " ( $\mathrm{T}$. Gerrard \& Co. Ltd., London) is suitable, and does not prevent access of the acid to the tissue.

After exposure to the acid all tissue elements show a great affinity for basic dyes, so that it is necessary to use dilute solutions of stain. In our experience $0.01 \%$ "azur A" gives excellent results, but in view of the well-known variation in staining qualities of different batches of metachromatic dyes, it is probably unwise to stipulate a definite dye concentration or staining time. These can be adjusted to individual preference, but we would caution against overstaining. Any dye which produces reasonably good metachromatic staining of such tissues as cartilage, umbilical cord, etc., should prove satisfactory if suitably diluted, but it is well to bear in mind that metachromasia of any tissue can be obscured by staining too intensely. The best preparations are those in which the background is stained a sky-blue, as then the metachromatic elements stand out most prominently. Should sections be overstained, the intensity of the colour can be reduced by rinsing them in $1 \%$ hydrochloric acid in $\mathbf{7 0} \%$ ethanol.

The metachromatic colour is highly stable. Prolonged exposure of stained sections to absolute ethanol, or to $1 \%$ hydrochloric acid in $70 \%$ ethanol, sufficient to discharge the blue completely, merely results in some reduction in the intensity of the metachromatic colour, but does not reverse the reaction.

\section{Results}

Sections stained by this method show beautiful polychrome effects, the colours ranging from bright red through purple, dark blue and pale blue, to green. Virtually all tissue elements capable of giving a positive periodic acid-Schiff reaction (McManus, 1948), e.g., mucins, basement membrane and reticulin, the brush borders of renal tubular and intestinal epithelium, thyroid colloid, exhibit intense metachromasia. A notable exception is glycogen, which does not give the reaction in formol-fixed tissues. Most cellular structures 
which are normally basophilic stain deep blue, whereas most acidophil tissue elements appear pale blue. Erythrocytes stain green, the negative metachromasia of Lison and Mutsaars (1950). The capacity of such tissue elements as cartilage or mast cells to stain metachromatically is not impaired by the acid treatment.

The most useful application of the method should be in the study of pathological conditions affecting the renal vasculature and interstitial tissues, as the glomerular capillary tufts and tubular basement membranes are revealed with great clarity. Even in avascular glomeruli which have become hyalinized the obliterated capillary loops are often recognizable in the hyaline.

The method has also proved useful in the study of sections of gastric mucosa. Both intra- and extra-cellular mucin exhibit intense metachromasia, while the zymogen granules of the chief cells appear deep blue. The epithelial basement membranes stain a reddish-purple, and the amorphous ground substance of the stroma appears pale pink.

The distinction between reticulin and collagen is emphasized. Thus, while the reticulins of spleen, lymph nodes, muscle, pituitary, adrenal, etc., as well as the basement membranes of kidney and glandular acini show well-marked metachromasia, collagen remains orthochromatic. This difference in reaction between reticulin and collagen is well shown in sections of ovary. The results obtained are very similar to those produced by the "Allochrome" modification of the periodic acidSchiff reaction introduced by Lillie (1951).

\section{Discussion}

Comparison of sections stained by the sulphuric acid-metachromatic reaction with controls treated by the McManus (1948) periodic acid-Schiff reagent method shows that virtually all tissue elements which give a periodic acid-Schiff reaction, with the exception of glycogen, exhibit intense metachromasia after treatment with concentrated sulphuric acid. Conversely, no sulphuric acid metachromatic-positive tissue components have been encountered which do not give a positive periodic acid-Schiff reaction. There is therefore little doubt that substances containing carbohydrate are responsible for the reaction.

A detailed study of the mechanism of the res action is at present in progress, and until this 19 completed we do not feel that histochemic $\vec{\Phi}$ specificity can be claimed for the method. It is. not proposed to embark on a detailed theoreticas discussion of the various possible reaction products. which might result from the action of sulphuris acid on carbohydrates. Preliminary investigationsis which, when completed, will be published in detaif give good reason to believe that the effect is due to the formation of sulphuric acid esters. This accords well with the known capacity of these substances to stain metachromatically.

Although, therefore, histochemical specificity fof the reaction is not claimed at present, the histo ${ }^{\circ}$ logical merits of the method justify its publicationo at this stage.

\section{Summary}

Certain tissue elements are capable of staining metachromatically after pre-treatment with concene trated sulphuric acid.

The reaction appears to be confined to substance\& capable of giving a positive periodic acid-Schiff reaction, suggesting that the metachromatic coloun is due to the presence of carbohydrate.

The distinction between a positive and negative. reaction is clearer than with the periodic acid-Schiff test, and the method is less time-consuming.

Our thanks are due to Dr. A. H. T. Robb-Smith. for his encouragement and for making the facilities oB his department available to us.

\section{REFERFNCES}

Lillie, R. D. (1951). Amer. J. clin. Path., 21, 484

Lison, L., and Mutsaars, W. (1950). Quart. J. micr. Sci., 91, 309. McManus, J. F. A. (1948). Stain Technol., 23, 99. - and Mowry, R. W. (1952). Lab. Invest., 1, 208. 